\title{
Using Virtual Reality to Experience Different Powered Wheelchair Configurations
}

\author{
Thomas W. Day ${ }^{1}$ and William H. Dobson ${ }^{1}$ and Christopher J. Headleand ${ }^{2}$ \\ and Nigel W. John ${ }^{1}$ and Serban R. Pop ${ }^{1}$
}

\begin{abstract}
This paper presents recent additions to our Wheelchair-VR application, in particular the use of different drive configurations. We have previously shown that Wheelchair-VR can be used to improve driving skills. Here we consider the utility of the application in allowing users who are in the process of purchasing or upgrading a wheelchair to experience different configurations and options in a costeffective virtual environment. A preliminary study is presented, which suggests that this approach can be effective.
\end{abstract}

\section{INTRODUCTION}

The recent availability of low cost, high quality Head Mounted Displays (HMDs) such as the Oculus Rift and HTC VIVE has stimulated a resurgence of interest in virtual reality (VR) applications. The accessibility of the technology is making possible new uses in a variety of different domains, not just entertainment applications. In previous work we have created a virtual environment called Wheelchair-VR for training driving skills for users of powered wheelchairs [9]. The user wears a HMD and is immersed in an environment where they are sitting in a virtual wheelchair. They then use a joystick to control the movement of the wheelchair and have to navigate through a series of different obstacles. A validation study has proved that this virtual environment is effective in training the driving skills needed for a real powered wheelchair [11].

In this paper we report on work to extend our original wheelchair simulator. Our hypothesis is that VR can be used to help a new user to train with different wheelchair specifications to help decide the optimum model for their particular needs (a virtual prototyping scenario). Factors that need to be taken into account are any restrictions from their disability, the layout of their home and workplace, and how often they are out of doors (traveling to work, shopping, visiting friends, restaurants, etc.). Our goal is to recreate in VR the scenarios that the user would regularly encounter, for example, using a pedestrian light controlled crossing to get across a busy road. They can then practice driving in that scenario using a virtual wheelchair that can be configured to behave with a variety of different schema, front-wheel drive, rear-wheel drive, etc. Typically expert advice on wheelchair choices will come from the assessment centres, with some information available in leaflets or online. In the UK, the

\footnotetext{
${ }^{1}$ Thomas W. Day, William H. Dobson, Nigel W. John, and Serban R. Pop are with the Department of Computer Science, part of the Faculty of Science and Engineering at the University of Chester nigel.john@chester.ac.uk

${ }^{2}$ Christopher J. Headleand is with the School of Computer Science, University of Lincoln cheadleandelincoln.ac.uk
}

Research Institute for Consumer Affairs (RICA) is a charity that focus specifically on issues of concern to disabled and older consumers. They have produced a useful guide to help choose a wheelchair [16], which has been the inspiration for the work presented in this paper. Our aim is to supplement the guide with VR tools that allow the user to experiment with the different wheelchair options that are described in the guide. This is particularly aimed at users with non-specialist needs or are experienced enough to know what they need.

Section 2 provides a brief background on related work in VR to help train individuals in performing a particular task, as well as the gamification of therapetic activities. In particular, we provide a summary of related work in wheelchair simulators. Section 3 the describes new features that we have added to our wheelchair simulator. This includes the different drive mechanisms, an outdoor scene with audio effects, and the use of hand tracking to allow the wheelchair user to interact with objects in the scene. We then present initial results from our system being used by able bodied volunteers and end with conclusions and future work.

\section{BACKGROUND}

\section{A. Training in Virtual Reality}

VR training provides a number of benefits, most notably that potentially dangerous scenarios can be simulated, without the associated risks. It also allows activities to be repeated and recorded precisely, allowing learners to reflect on training experiences, develop new ideas, and test them in practice. VR provides a potentially ideal environment for experiential learning [12]. Furthermore, it provides a way of ensuring consistency in delivery of specific protocols [1]. For reasons such as these, VR has been explored as an educational tool since the inception of the technology. However, early investigations [13] indicated that skills transfer from VR to the real world is limited. Research largely blamed the quality of the VR technology of the time; until recently focus shifted to other virtual world technologies such as CAVE environments.

However, later efforts, such as the ones from Loftin and Kenney [14], [18] and Seymour et al. [17] demonstrated positive results, regarding skills transfer. The recent launch of many cost effective VR devices is likely to support a continuation of this trend.

\section{B. Gamification of Therapeutic Activities}

There are a number of reasons why we may wish to use simulation as a wheelchair training tool. Beyond the 
possible financial and accessibility benefits that simulation could provide, a virtual world also provides numerous playful opportunities. Gamification is known to have a number of positive attributes [6], and this is something we can harness.

There are a number of examples where games have been used to improve quality of life. For example, older adults often have a lifestyle of reduced activity which can have negative implications for their health and life expectancy. Motion controlled games have been used as a tool to encourage a positive engagement with physical exercise despite age-related impalements. An excellent discussion of this area including guidelines for the design of full-body motion-based games for older adults is provided by Gerling et al. [4]. VR-based games have also been applied to this challenging area [15], this includes the training and reinforcement of motor control skills through dancing [1].

While game-based engagements are often associated with a younger audience, these studies show that they are suitable and engaging for older adults also. As a large number of wheelchair users are older adults with motion impairments, these positive results support ongoing research.

Specific wheelchair-based video games have been shown to be engaging for a varied demographic of users [3], [5]. There is also evidence that games-based experiences can help users develop a positive relationship with the wheelchair [5].

\section{Wheelchair Simulators}

Early work by Hasdai et al. demonstrated the training benefit of wheelchair simulators [8]. New wheelchair users who had trained on the simulator were significantly better drivers than the control group who had not. However, both groups were outperformed by a group of experienced wheelchair users.

Another early example focused on young users [10], specifically explored entertaining environments that could motivate children. The results demonstrated that driving skill could be improved through a simulator. While this simulator has a VR modality, the users preferred to use a monitor, though it is worth noting that the VR equipment of the era was relatively low quality compared to the current state-ofthe-art. For this reason as well as cost, much of the early work in this field focused on non-immersive environments. However, other technical limitations were encountered. For example, Desbonnet et al. [2] note that visual realism and the behaviour of their virtual wheelchair was not sufficient.

More recently, Harrison et al. [7] explored a nonimmersive wheelchair simulator using a computer monitor with 6 participants. While the results of the study were largely positive, the authors note that the virtual environment was too challenging, and that this must be addressed for the simulator to be a motivating training tool.

\section{Methods And TOOLS}

Wheelchair-VR has been developed using the Unity 3D game development platform (Unity Technologies, San Francisco, CA). The user interface consists of the Oculus Rift HMD to immerse the user within the virtual environment, with headphones for sound effects. One of the thumbsticks on a Microsoft XBox controller is used as the wheelchair joystick for selecting the direction of motion, and any direct interaction from the user's hands is achieved with the Leap Motion (Leap Motion Inc., CA, USA) hand tracking device. Figure 1 shows the system in use.

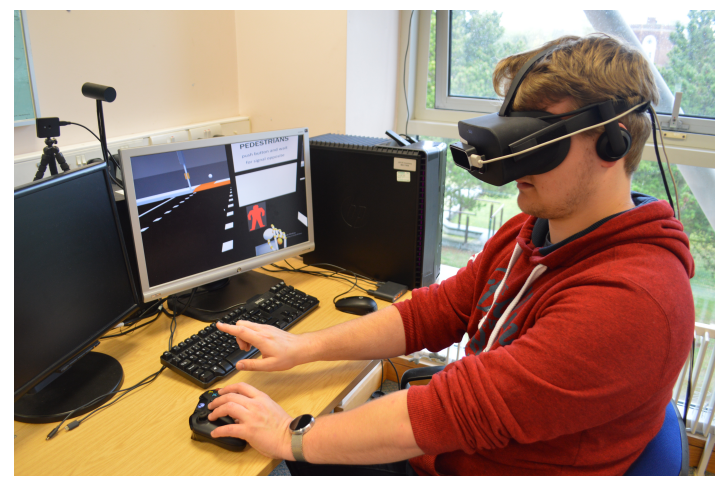

Fig. 1. Wheelchair-VR in use. Note Leap Motion connected to Oculus Rift and XBox controller joystick for controlling the virtual wheelchair.

\section{A. Simulated chair}

The simulated chair can function as either a Class 2 wheelchair (can only be used on the pavement and limited to traveling at $4 \mathrm{mph}$ ) or a Class 3 wheelchair (can be used on the pavement at up to $4 \mathrm{mph}$, or on the road at up to $8 \mathrm{mph}$ ). Class 3 wheelchairs require certain extra safety features such as indicators and a horn. The user is given the option to choose which Class they want to use and the maximum speed of the simulated chair is limited accordingly within the Unity motion control scripts.

Audio samples of real powered wheelchairs were collected and edited to reduce noise and set amplification levels. These samples were then be attached as an audio source to the base of the virtual wheelchair with a corresponding audio listener attached to the first person camera. A unity script was written to play the appropriate wheelchair sound dependent on the action being performed by the user e.g., if the user moves forward on the joystick then a wheelchair accelerating sound is played. An audio mixer component also allows the volume to change with the speed of the wheelchair.

\section{B. Wheelchair Drive Configurations}

The movement of the chair is based on a kinematic character controller. Character controllers are designed to produce physics-like interactions without needing to accurately simulate physics. This has a number of advantages over a pure physics approach. Firstly, physically simulated movement is the combination of a variety of parameters such as torque, friction, mass, weight-distribution, and drag. This makes fine-tuning implementations complex, as a small change in one parameter can have a drastic effect on another. This is further complicated as a game engine's physics engine only produces an approximation of physicality, so an accurate model may still not produce the outcome you expect. Using a kinematic model allows realistic movement to be produced 
without having to precisely model these characteristics. An additional benefit of this approach is that we can abstract different aspects of the chair into a number of largely self-contained, logical parameters. Adjusting these allows us to faithfully recreate a number of different wheelchair modalities.

The parameters used in the wheelchair are:

- Slope Limit: a setting which determines the maximum incline that the chair be driven up. Many chair manufactures provide some guidance on the slopes their chairs can successfully navigate which can be incorporated into this parameter.

- Pivot Center: a position which determines where the chair pivots from during a turn. By moving the pivot point forward or aft allows for the simulation of rear, and center wheel drive chairs;

- Acceleration: a curve which determines the characteristics of how the chair accelerates between 0 and maximum speed.

- Turn Speed: a curve which determines how quickly the chair rotates during a turn.

- Stopping Speed: determines how quickly the chair stops once the user stops applying power.

While this study makes use of a generic rear, centre, and front wheel drive chair, the modification of these parameters allows all motorized wheelchairs to be simulated.

\section{Driving Scenarios}

The intention is to provide a library of different scenarios, some of which can be customisable. For example, to recreate the furniture layout in the users own lounge. However, for our initial work we have focused on two tasks that require good wheelchair maneuverability: a maze built from kerbstones, where the user must avoid collisions with the kerb (Figure 2 ); and an outdoor scene where the user has to drive across a road using a pedestrian crossing. Both scenes were built using the Unity editor - see Figure 3.

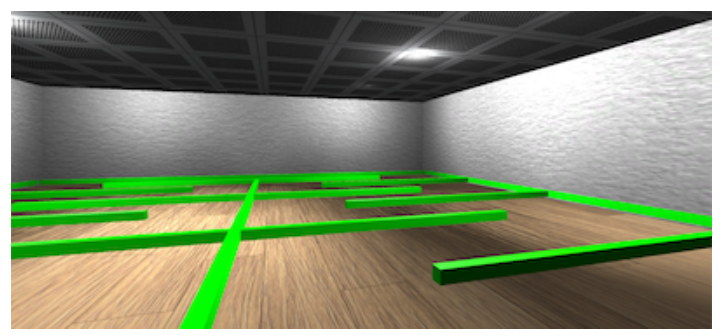

Fig. 2. Kerbstone maze - kerb colour changes from green to red and a sound effect is played if the wheelchair collides with it.

Car models were also introduced into the outdoor scene and were scripted to accurately control car movement in their respective lanes and how they would react to the changing traffic signals. The traffic lights are triggered in the scene when the wheelchair user reaches out to touch the crossing button. To achieve this the Leap Motion device has been attached to the HMD. It uses optical sensors and infrared light to track hand movements and a Unity plugin is available

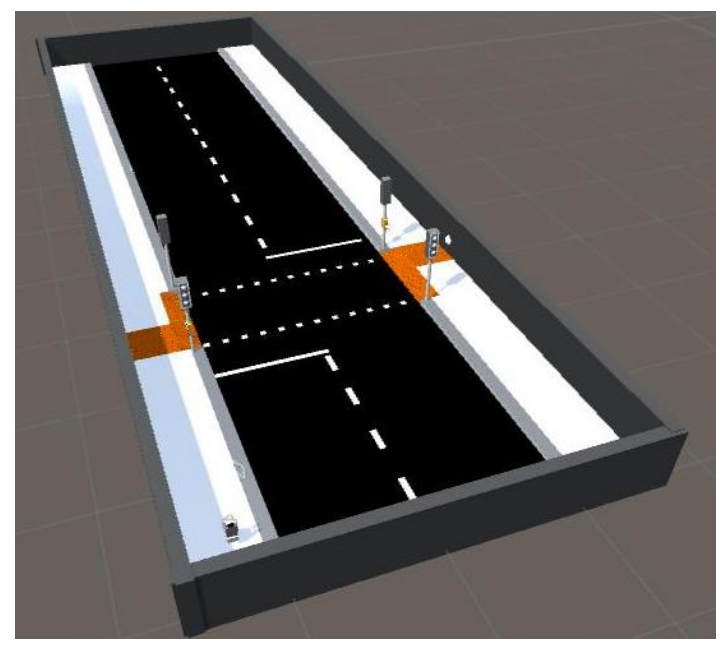

Fig. 3. Pedestrian Crossing layout in Unity Editor. All measurements are based on information available in the Highway Code, a set of information, advice, guides and mandatory rules for all road users in the United Kingdom.

so that this information can be accessed from within a Unity scene. Scripts included with the plugin translate Leap Motion coordinates to the Unity coordinate system. Once the button is selected, the traffic lights will change to red after a short delay and the green crossing man will appear, together with an audio cue that it is safe to cross - see Figure 4. The virtual cars will stop at the lights. After a standard amount of time, the lights will change back to green and the cars will continue.

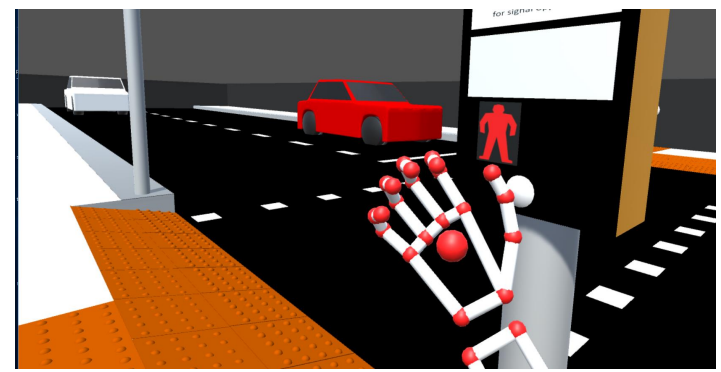

Fig. 4. The Leap Motion allows a user's hand to be tracked, used here to press the button on the crossing lights

\section{RESULTS}

A pilot study has been carried out to test the effectiveness of our application as a tool for an individual to try out different wheelchair drive mechanisms and to identify where further improvements are needed. Ten able-bodied participants were used, none of whom were experienced users of powered wheelchairs. They had to use WheelchairVR to navigate through two different scenarios three times, once each with a rear, centre and front wheel drive systems. The first scenario involved navigating from one side of the room to the other through a maze of kerbstones (Figure 2). The second scenario involved traveling along a pavement to the pedestrian crossing, pressing the crossing button (Figure 4), and then getting across the road once the green 
man is displayed. Participants were not told what type of wheelchair they were using, just wheelchair A, B and C. The wheelchair order was randomised for each participant. Time taken was recorded and on completion everyone filled in a questionnaire where they were asked to rate which system they preferred for each task, which system they thought the best for turning, and any other comments they had.

The average time for completing the first scenario was: Centre, 81 seconds (standard deviation 0.11); Front, 77 seconds (SD 0.11); and Rear, 73 seconds (SD 0.07). The average time for completing the second scenario was: Centre, 48 seconds (SD 0.2); Front, 44 seconds (SD 0.07); and Rear, 41 seconds (SD 0.04). The choice of preferred system was mixed, but there was a slight majority for the Centre drive system for the first scenario, and an equal preference for the Centre and Front systems in the second scenario. The Centre drive system was also chosen the most as the best for turning.

A majority of the participants also experienced some discomfort whilst using Wheelchair-VR, and reported typical symptoms of cybersickness.

\section{DisCUSSION AND CONCLUSIONS}

According to the RICA guide [16]:

- Centre drive is the most manoeuvrable as it minimises the wheelchairs turning circle. Six wheels also help maintain stability on slopes. It can give a bumpy ride on uneven ground, as the drive wheels are right under the seat so is not usually recommended for outdoor use.

- Front drive is best for steering around tight corners and use on uneven terrain, but can be more difficult to steer at high speed. It is also usually more stable on slopes.

- Rear is the most common system. The turning circle is larger than other systems, but many people find it easier to drive in a straight line.

Whereas all of the participants in our pilot study could detect a difference in the behaviour of the drive systems their preferences only partly reflect the expected performance according to the above. The Centre system was preferred for the first scenario, and rated the best for turning. The Centre system was preferred for the first scenario, and rated the best for turning. Note that the average time to complete the task is slower when using the Centre drive system. This does not necessarily mean it is harder to use, as driving the chair more slowly helps to successfully navigate sharp corners.

The average time to complete the second scenario was roughly the same for all three chairs. Participants tended to prefer either the Centre of Front drive systems. However, a problem with the second scenario is that it does not currently replicate the bumps and unevenness of a typical pavement. This needs to be simulated - possible with the use of a motion platform - to allow a better fidelity experience for outdoor use. It is likely then that the Centre drive would be more uncomfortable and more participants would prefer the Front drive mechanism - which is good for tight corners and uneven terrain.

With only ten participants this pilot study is too small to obtain any significant results. However, the results do indicate that Wheelchair-VR can allow users to experiment with different wheelchair configurations and support the validity of our hypothesis. We will improve the software before conducting a larger scale study. Simulation of uneven terrain, including scenarios with more slopes, and reducing the effects of cybersickness are all currently being addressed.

\section{REFERENCES}

[1] E.D. de Bruin, D. Schoene, G. Pichierri, and S.T. Smith. Use of virtual reality technique for the training of motor control in the elderly. Zeitschrift für Gerontologie und Geriatrie, 43(4):229-234, 2010.

[2] M Desbonnet, S Cox, and A Rahman. Development and evaluation of a virtual reality based training system for disabled children. In Proceedings of the European Conference on Disability, Virtual Reality and Associated Technologies, volume 11, pages 177-182, 1998.

[3] Kathrin Gerling, Kieran Hicks, Michael Kalyn, Adam Evans, and Conor Linehan. Designing movement-based play with young people using powered wheelchairs. In Proceedings of the 2016 CHI Conference on Human Factors in Computing Systems, pages 4447-4458. ACM, 2016.

[4] Kathrin Gerling, Ian Livingston, Lennart Nacke, and Regan Mandryk. Full-body motion-based game interaction for older adults. In Proceedings of the SIGCHI Conference on Human Factors in Computing Systems, pages 1873-1882. ACM, 2012.

[5] Kathrin M Gerling, Regan L Mandryk, and Michael R Kalyn. Wheelchair-based game design for older adults. In Proceedings of the 15th International ACM SIGACCESS Conference on Computers and Accessibility, page 27. ACM, 2013.

[6] Juho Hamari, Jonna Koivisto, and Harri Sarsa. Does gamification work?-a literature review of empirical studies on gamification. In System Sciences (HICSS), 2014 47th Hawaii International Conference on, pages 3025-3034. IEEE, 2014.

[7] A Harrison, G Derwent, A Enticknap, F Rose, and E Attree. The role of virtual reality technology in the assessment and training of inexperienced powered wheelchair users. Disability \& Rehabilitation, 24(11):599-606, 2002.

[8] Aya Hasdai, Adam S Jessel, and Patrice L Weiss. Use of a computer simulator for training children with disabilities in the operation of a powered wheelchair. American Journal of Occupational Therapy, 52(3):215-220, 1998.

[9] Christopher J Headleand, Thomas Day, Serban R Pop, Panagiotis D Ritsos, and Nigel W John. A cost-effective virtual environment for simulating and training powered wheelchairs manoeuvres. pages 134141. IOS Press, 2016.

[10] D Inman, K Loge, and J Leavens. VR Education and Rehabilitation. Communications of the ACM, 40(8):53-58, 1997.

[11] Nigel W John, Serban R Pop, Thomas W. Day, Panagiotis D. Ritsos, and Christopher J. Headleand. The implementation and validation of a virtual environment for training powered wheelchair manoeuvres. IEEE Transactions on Visualization and Computer Graphics, In Press, 2017.

[12] David A Kolb. Experiential learning: Experience as the source of learning and development. FT press, 2014.

[13] JJ Kozak, PA Hancock, EJ Arthur, and ST Chrysler. Transfer of training from virtual reality. Ergonomics, 36(7):777-784, 1993.

[14] R. B. Loftin and P. Kenney. Training the hubble space telescope flight team. IEEE Computer Graphics and Applications, 15(5):31-37, Sep 1995.

[15] Karina Iglesia Molina, Natalia Aquaroni Ricci, Suzana Albuquerque de Moraes, and Monica Rodrigues Perracini. Virtual reality using games for improving physical functioning in older adults: a systematic review. Journal of NeuroEngineering and Rehabilitation, 11(1):156, 2014.

[16] RICA. Getting a powered wheelchair, a guide to help you choose. Brochure, 2015.

[17] Neal E Seymour, Anthony G Gallagher, Sanziana A Roman, Michael K Obrien, Vipin K Bansal, Dana K Andersen, and Richard M Satava. Virtual reality training improves operating room performance: results of a randomized, double-blinded study. Annals of surgery, 236(4):458-464, 2002.

[18] Paul N. Wilson, Nigel Foreman, and Michael Tlauka. Transfer of spatial information from a virtual to a real environment. Human Factors, 39(4):526-531, 1997. 